\title{
Phytoprotection
}

\section{Diversité et abondance des pucerons [Homoptera : Aphididae] et leur impact sur la dissémination des virus infectant la pomme de terre au Mali \\ Diversity and abundance of aphids [Homoptera: Aphididae] and their impact on the spread of viruses infecting potato in Mali}

\author{
Almouner A.A. Yattara, Amadou K. Coulibaly et Frédéric Francis
}

Volume 94, numéro 1, 2014

Reçu le 2013-05-13; accepté le 2013-09-23

URI : https://id.erudit.org/iderudit/1024719ar

DOI : https://doi.org/10.7202/1024719ar

\section{Aller au sommaire du numéro}

\section{Éditeur(s)}

Société de protection des plantes du Québec (SPPQ)

ISSN

1710-1603 (numérique)

Découvrir la revue

Citer cet article

Yattara, A. A., Coulibaly, A. K. \& Francis, F. (2014). Diversité et abondance des pucerons [Homoptera : Aphididae] et leur impact sur la dissémination des virus infectant la pomme de terre au Mali. Phytoprotection, 94(1), 1-7. https://doi.org/10.7202/1024719ar

\section{Résumé de l'article}

Des études sur l'abondance et la diversité des pucerons ont été menées pendant trois campagnes agricoles au Mali. Sur la base de relevés de bacs jaunes installés dans des cultures de pomme de terre à Kati et à Sikasso, 2525 pucerons ont été capturés et identifiés. Dix-neuf espèces de pucerons ont été recensées, dont deux qui ont été observées in situ sur la culture : Aphis gossypii (Glover) et Myzus persicae (Sulzer). La plupart de ces espèces sont des ravageurs de cultures et elles contribuent également à la transmission virale. Des échantillons foliaires prélevés dans des parcelles de pomme de terre dans les deux régions ont été testés par la technique ELISA pour la détection des deux principaux virus dommageables, soit le Potato Virus Y (PVY) et le Potato Leaf Roll Virus (PLRV). Le taux de plantes virosées dans les deux localités pendant les trois années variait de 19,3 \% à 21,8 \% pour le PVY, alors qu'il était de $8,5 \%$ à 9,3\% pour le PLRV. L'occurrence de ces maladies virales s'est révélée être très homogène d'une année à l'autre, avec des taux relativement importants. Cette étude est une première quantification dans cette région du Mali de l'importance des relations pucerons vecteurs-virus en culture de pomme de terre.
Tous droits réservés @ La société de protection des plantes du Québec, 2014
Ce document est protégé par la loi sur le droit d'auteur. L’utilisation des services d'Érudit (y compris la reproduction) est assujettie à sa politique d'utilisation que vous pouvez consulter en ligne.

https://apropos.erudit.org/fr/usagers/politique-dutilisation/ 


\title{
Diversité et abondance des pucerons [Homoptera: Aphididae] et leur impact sur la dissémination des virus infectant la pomme de terre au Mali
}

\author{
Almouner A.A. Yattara ${ }^{1,2} \bowtie$, Amadou K. Coulibaly $^{2}$ et Frédéric Francis ${ }^{1}$ \\ Reçu le 2013-05-13; accepté le 2013-09-23
}

PHYTOPROTECTION $94: 1-7$

Des études sur l'abondance et la diversité des pucerons ont été menées pendant trois campagnes agricoles au Mali. Sur la base de relevés de bacs jaunes installés dans des cultures de pomme de terre à Kati et à Sikasso, 2525 pucerons ont été capturés et identifiés. Dix-neuf espèces de pucerons ont été recensées, dont deux qui ont été observées in situ sur la culture: Aphis gossypii (Glover) et Myzus persicae (Sulzer). La plupart de ces espèces sont des ravageurs de cultures et elles contribuent également à la transmission virale. Des échantillons foliaires prélevés dans des parcelles de pomme de terre dans les deux régions ont été testés par la technique ELISA pour la détection des deux principaux virus dommageables, soit le Potato Virus Y (PVY) et le Potato Leaf Roll Virus (PLRV). Le taux de plantes virosées dans les deux localités pendant les trois années variait de 19,3\% à 21,8 \% pour le PVY, alors qu'il était de $8,5 \%$ à $9,3 \%$ pour le PLRV. L'occurrence de ces maladies virales s'est révélée être très homogène d'une année à l'autre, avec des taux relativement importants. Cette étude est une première quantification dans cette région du Mali de l'importance des relations pucerons vecteurs-virus en culture de pomme de terre.

Mots-clés: Abondance, aphidifaune, diversité, Kati, Mali, pomme de terre, PLRV, PVY, Sikasso.

[Diversity and abundance of aphids [Homoptera: Aphididae] and their impact on the spread of viruses infecting potato in Mali]

Studies on the abundance and diversity of aphids were conducted during three crop seasons in Mali. Based on the survey of yellow traps set in potato crops in Kati and Sikasso, 2,525 winged aphids were collected and identified. Nineteen species of aphids were identified, two of which were also observed in situ on potato plants: Aphis gossypii (Glover) and Myzus persicae (Sulzer). Most of these species are crop pests and they also contribute to viral transmission. Leaf samples taken in potato plots in both regions were tested by ELISA for the detection of two major viruses: Potato Virus Y (PVY) and Potato Leaf Roll Virus (PLRV). The rate of virus-infected plants at both locations during the three years ranged from $19.3 \%$ to $21.8 \%$ for PVY, and from $8.5 \%$ to $9.3 \%$ for PLRV. The occurrence of these viral diseases was very consistent from one year to the next, with relatively high levels. This study is the first survey conducted in this region of Mali on the importance of aphids and associated viruses in potato fields.

Keywords: Abundance, aphidifauna, diversity, Kati, Mali, PLRV, potato, PVY, Sikasso.

1. Unité d'entomologie fonctionnelle et évolutive, Gembloux Agro-Bio Tech, Passage des Déportés, 2.B-5030, Université de Liège, Belgique; $\triangle$ pedrosannas@yahoo.fr

2. Laboratoire de Biologie des Arthropodes et de Lutte Intégrée, IPR/IFRA de Katibougou, Mali 


\section{INTRODUCTION}

La pomme de terre (Solanum tuberosum L.) est I'une des premières ressources alimentaires au monde. Elle est cultivée aussi bien dans les régions tempérées que dans les régions tropicales ou équatoriales (Rolot 2005). Cette culture est sujette à de nombreuses agressions, parmi lesquelles les pucerons occupent une place importante. II s'agit d'un groupe $d^{\prime}$ 'insectes phytophages très important, aussi bien par le nombre d'espèces (4 700 taxa de 600 genres), que par leur biologie et la nature des dégâts qu'ils occasionnent (Remaudière et Remaudière 1997). Environ 450 espèces aphidiennes ont été identifiées comme ravageurs de plantes cultivées (Blackman et Eastop 2000). Parmi elles, une centaine s'est adaptée à divers agro-écosystèmes et présente, de ce fait, une importance économique notable (Blackman et Eastop 2007). Au moins 220 espèces ont été recensées en Afrique, au sud du Sahara (Autrique et Ntahimpera 1994). Le réchauffement climatique est régulièrement cité pour expliquer les changements de dispersion géographique des pucerons. En moyenne, huit espèces apparaissent à chaque degré Celsius supplémentaire dans une région ciblée (Harrington et al. 2007). Ces insectes sont vecteurs de nombreux virus. À l'échelle mondiale, six virus de la pomme de terre sont considérés comme majeurs et économiquement dommageables (Marchoux et al. 2008). En Afrique tropicale, notamment au Mali, nous ne disposons que de peu d'information quant au nombre de viroses transmises par les pucerons. Toutefois, les principaux groupes de vecteurs du monde sont présents sur ce continent, certainement avec des importances relatives différentes. Les pucerons sont partout les vecteurs majoritaires pour de nombreuses maladies virales transmises à une large proportion de plantes (Fauquet et Thouvenel 1987).

Au Mali, la pomme de terre est principalement cultivée dans les zones spécifiques de Sikasso et Kati (Sidibé et al. 2008), surtout pendant la saison sèche-fraîche d'octobre à mars. À Kati, la pomme de terre est cultivée autour des points d'eau, en culture maraîchère en association, entre autres, avec le chou (Brassica spp.), la tomate (Lycopersicum spp.), le tabac (Nicotiana tabacum L.), le poivron (Capsicum spp.) et I'oignon (Allium spp.), alors qu'à Sikasso, elle est généralement cultivée en monoculture (après le riz [Oryza spp.]) dans les bas-fonds dotés d'une nappe phréatique proche de la surface. Les producteurs de pomme de terre sont fortement dépendants des semences importées provenant d'Europe, principalement de la France, constituées de variétés comme 'Sahel', 'Claustar', 'Élodie' et 'Pamina'. Plusieurs espèces d'adventices pouvant servir d'hôtes aux pucerons ont également été observées dans et autour des champs. Quelques virus ont été signalés sur les cultures de pommes de terre au Mali. Ainsi, à Sikasso, la présence du virus $S$ (PVS), du virus $X(P V X)$ et du virus $Y(P V Y)$ de la pomme de terre a été mentionnée (Coulibaly et al. 2002). Les symptômes du virus de l'enroulement de la pomme de terre (PLRV) ont parfois été observés sans toutefois qu'il y ait de diagnostic confirmatif. Parmi les différents facteurs limitant la production de la pomme de terre au Mali, notamment à Kati et à Sikasso, les virus semblent être des contraintes majeures. Bien qu'il n'y ait pas de rapport officiel sur la présence de virus dans les zones de production faisant l'objet de l'étude, la présence des principaux insectes ravageurs vecteurs de viroses sur la pomme de terre est rapportée. En revanche, aucune information n'est disponible quant à leur rôle dans la dissémination potentielle de viroses dans la zone, et ce, malgré la large gamme d'espèces potentiellement vectrices (Hobbs et al. 2000; Sikora et al. 1998).

Des études sur la diversité et l'abondance des pucerons et des virus associés constituent une étape importante dans le développement d'outils de diagnostic et de mesures de contrôle appropriées. L'objectif de cette étude est d'évaluer la diversité et l'abondance des pucerons dans les parcelles de pomme de terre à Kati et Sikasso, au Mali, afin d'avoir une idée de leur impact potentiel dans la dissémination des viroses et de mettre à la disposition des acteurs de lutte contre les viroses de la pomme de terre une base de données nécessaire dans la gestion des insectes vecteurs dans un contexte de changements climatiques.

\section{MATÉRIEL ET MÉTHODES}

Cette étude a été réalisée à Kati $\left(12^{\circ} 44^{\prime} 48^{\prime \prime} \mathrm{N}\right.$ et $\left.8^{\circ} 4^{\prime} 17^{\prime \prime} \mathrm{E}\right)$ et à Bamadougou, près de Sikasso $\left(10^{\circ} 55^{\prime} 0^{\prime \prime} \mathrm{N}\right.$ et $7^{\circ} 0^{\prime} 0^{\prime \prime}$ E), à l'ouest et au sud du Mali, respectivement. Un total de neuf parcelles de pomme de terre de consommation ont été choisies (quatre à Kati et cinq à Sikasso) de manière à inclure différentes conditions climatiques et géographiques.

\section{Collecte des pucerons}

Deux méthodes ont été combinées pour la surveillance de I'aphidifaune: I'installation de pièges jaunes de Von Moericke $(\varnothing: 27 \mathrm{~cm}, \mathrm{~h}: 10 \mathrm{~cm})$ et des observations visuelles in situ. Ce type de piège est I'un des modèles les plus fréquemment utilisés en entomologie faunistique des milieux agricoles, car il est efficace et se prête à des échantillonnages de grande envergure (Mignon et al. 2003). L'évaluation de la diversité et de l'abondance des espèces de pucerons a été effectuée en collectant les insectes capturés dans des bacs jaunes installés dans un réseau de quatre parcelles à Kati et de cinq à Sikasso. La superficie des parcelles variait entre 1 et 3 ha. Environ 1 mo après le semis, trois pièges jaunes ont été installés en triangle de $10 \mathrm{~m}$ de côté au centre de la parcelle. À ce moment, les bacs sont remplis d'eau jusqu'au trait de jauge et suspendus à leur support à la hauteur du plant de pomme de terre. Un détergent est additionné à cette eau. L'eau est ajoutée régulièrement en périodes de fortes chaleurs et renouvelée lors des collectes. Un changement hebdomadaire du contenu du récipient est nécessaire pour obtenir un rendement optimum (EDES 2012), car les pièges colorés sont dépendants des conditions atmosphériques. Les relevés et les observations ont été effectués hebdomadairement de novembre à mars, durant trois saisons culturales successives (2009 à 2011). Les insectes prélevés ont été triés sous binoculaire afin d'extraire les pucerons. Les pucerons recueillis ont été conservés dans du norvanol à $70 \%$, comptés, puis identifiés jusqu'au rang taxonomique de l'espèce. 


\section{Observation visuelle des pucerons et collecte des échantillons foliaires}

Dans une diagonale de chaque parcelle, trois zones de $100 \mathrm{~m}^{2}$ ont été délimitées. L'estimation des pucerons aptères retrouvés sur les feuilles de pomme de terre a consisté à observer, dans chaque zone, 10 plants choisis au hasard; le comptage des insectes s'est fait par observation directe. Simultanément aux observations visuelles, 10 feuilles ont été prélevées dans chaque zone, le long du trajet (figure 1), à tous les $4 \mathrm{~m}$ environ. L'ensemble a été déposé dans un sachet clairement identifié (parcelle, $\mathrm{n}^{\circ}$ zone, date), en plus des feuilles montrant des symptômes du PVY et du PLRV. Les échantillons foliaires de pomme de terre ainsi prélevés dans les parcelles ont été mis à sécher au soleil pendant une journée, puis conservés dans des sacs en polyéthylène au réfrigérateur avant leur acheminement au laboratoire d'entomologie fonctionnelle et évolutive de Gembloux Agro-Bio Tech (EFEGXABT) pour l'analyse par test ELISA (enzyme-linked immunosorbent assay) ciblant principalement le PVY et le PLRV. La diversité des pucerons a été évaluée jusqu'au rang taxonomique de l'espèce, après l'identification au laboratoire d'EFE-GxABT. Pour ce faire, plusieurs clés de détermination systématique spécifique ont été utilisées. L'identification des pucerons a été réalisée en se basant sur des caractères morphologiques tels que décrits par Remaudière et al. (1985),
Leclant (1999), Jacky et Bouchery (1982), Autrique et Ntahimpera (1994), Taylor (1981), ainsi que sur des spécimens de référence conservés au laboratoire d'EFE-GXABT. La richesse spécifique et l'abondance relative de chaque espèce ont été calculées telles que définies par Barbault (1992).

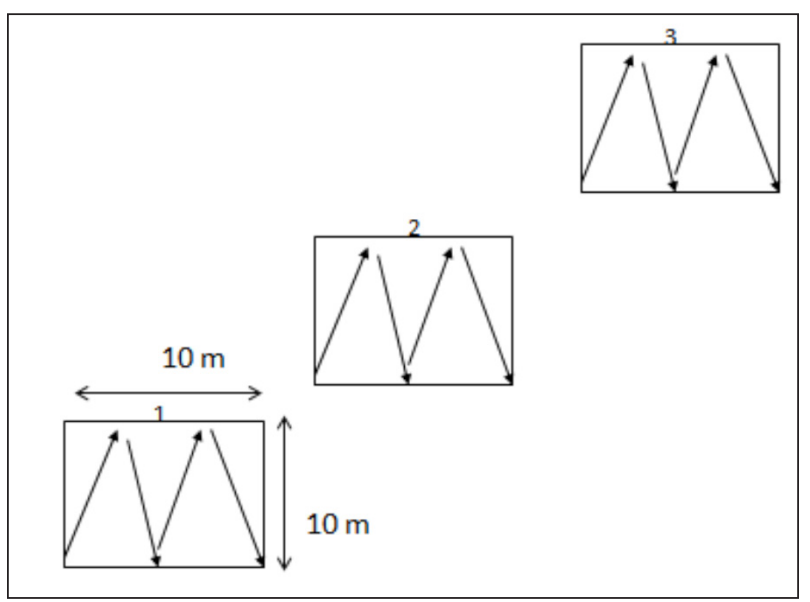

Figure 1. Dispositif d'échantillonnage des feuilles de pomme de terre dans les parcelles.

Tableau 1. Proportions et richesses relatives des pucerons piégés en cultures de pomme de terre de 2009 à 2011 au Mali.

\begin{tabular}{|c|c|c|c|c|c|c|}
\hline \multirow[b]{2}{*}{ Espèces de pucerons } & \multicolumn{3}{|c|}{ Kati } & \multicolumn{3}{|c|}{ Sikasso } \\
\hline & 2009 & 2010 & 2011 & 2009 & 2010 & 2011 \\
\hline Aphis craccivora (Koch, 1854) & $2,1^{*}$ & 1,0 & 3,6 & 0,6 & 2,5 & 0,6 \\
\hline Aphis fabae (Scopoli, 1763) & 2,9 & - & 2,2 & - & 0,4 & 2,4 \\
\hline Aphis gossypii (Glover, 1877) & 19,5 & 3,7 & 22,5 & 14,5 & 3,8 & 12,2 \\
\hline Brevicoryne brassicae (Linnaeus, 1758) & 1,7 & - & - & - & - & - \\
\hline Cinara cupressi (Buckton, 1881) & 0,4 & - & - & - & - & - \\
\hline Lypaphis erysimi (Kaltenbach, 1843) & 27,4 & 84,4 & 13,0 & 1,2 & 1,7 & 8,8 \\
\hline Melanaphis sacchari (Zehntner, 1897) & 0,4 & 0,8 & 0,7 & - & 0,4 & 2,6 \\
\hline Myzus persicae (Sulzer, 1776) & 21,2 & 2,7 & 39,1 & 22,5 & 0,4 & 23,4 \\
\hline Pentalonia nigronervosa (Coquerel, 1859) & - & 0,6 & 0,7 & - & - & 2,3 \\
\hline Pseudaphis sijui (Eastop, 1953) & - & 0,1 & - & - & - & - \\
\hline Rhopalosiphum insertum (Walker, 1849) & 1,7 & - & - & 0,6 & - & - \\
\hline Rhopalosiphum maidis (Fitch, 1856) & 5,4 & - & - & - & - & - \\
\hline Rhopalosiphum padi (Linnaeus, 1758) & 4,6 & 0,2 & - & 5,2 & 0,4 & - \\
\hline Rhopalosiphum rufiabdominalis (Sasaki, 1899) & 9,1 & 5,7 & 13,0 & 54,2 & 88,7 & 42,4 \\
\hline Sitobion graminis (Takahashi, 1950) & 0,8 & 0,3 & 2,3 & - & - & 2,3 \\
\hline Sitobion nigrinectaria (Theobald, 1915) & - & 0,1 & 0,7 & - & - & 1,6 \\
\hline Tetraneura nigriabdominalis (Sasaki, 1899) & - & 0,4 & 2,2 & - & 1,7 & 1,4 \\
\hline Tetraneura spp. (Hortig, 1841) & 0,8 & - & - & 0,6 & - & - \\
\hline Toxoptera aurantii (Boyer de Fonscolombe, 1841) & 2,0 & - & - & 0,6 & - & - \\
\hline Nombre total d'individus & 241 & 1226 & 138 & 173 & 238 & 509 \\
\hline Nombre d'espèces & 15 & 12 & 11 & 9 & 9 & 11 \\
\hline
\end{tabular}

* Les proportions sont exprimées en pourcentage. 


\section{Détection de virus}

La présence de virus a été vérifiée par test ELISA (DSMZ, Braunschweig, Allemagne) selon les méthodes DAS (double antibody sandwich) pour le PVY et TAS (triple antibody sandwich) pour le PLRV, comme décrit par Clark et Adams (1977), en suivant les instructions des fabricants. Le contrôle positif de la souche PVYNTN conservée sur Nicotiana tabacum cv. Xanthii (tabac) ainsi que le témoin négatif (plants sains de tabac) proviennent du laboratoire de phytopathologie de I'Université Catholique de Louvain (UCL, Belgique) et celui du PLRV (souche DSMZ $\mathrm{n}^{\circ} \mathrm{PV}$-0842) maintenu sur Solanum tuberosum var. Erna a été fourni par DSMZ. Les résultats ont été analysés à l'aide du logiciel Minitab, par la méthode de l'analyse de la variance (ANOVA).

\section{RÉSULTATS}

\section{Richesse et abondance relative des espèces de pucerons}

Durant l'étude, 2525 pucerons ont été collectés sur I'ensemble des deux sites à Kati et Sikasso pendant les campagnes 2009 à 2011. Près de deux tiers des captures $(63,56 \%)$ ont été réalisés à Kati. L'abondance des espèces varie selon les saisons et la région. Bien que l'abondance et la richesse spécifique des pucerons par rapport aux deux sites soient similaires (Tableau 1), le nombre d'espèces observées est faible, soit 19 espèces à Kati et 15 à Sikasso. Si I'on considère l'ensemble des sites, les espèces les plus abondantes au cours des trois campagnes sont, respectivement, Lypaphis erysimi (46,3\%), Rhopalosiphum rufiabdominalis (25\%), Myzus persicae (11,8\%) et Aphis gossypii (8,7\%).

\section{Observations visuelles des pucerons sur la pomme de terre}

Aphis gossypii et Myzus persicae ont été observés dans les parcelles de pomme de terre durant les trois années. Les abondances relatives par zone de production et par an sont présentées à la figure 2. Les plantes infestées sont généralement celles qui souffrent d'un stress hydrique. Si des variations importantes de

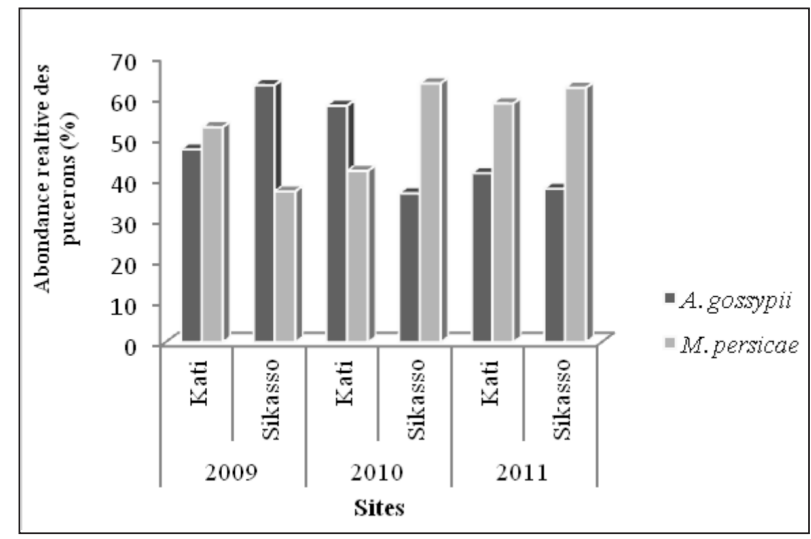

Figure 2. Abondances relatives de deux espèces de pucerons observés dans les parcelles de pomme de terre en fonction des sites (Kati et Sikasso) et des années. dominance sont observées durant les deux premières années, une certaine similarité d'abondance relative est uniquement constatée sur les deux sites en 2011.

En considérant la moyenne de trois années d'observation, la dynamique de population des deux espèces montre une apparition plus précoce de $M$. persicae dès les premières semaines sur l'ensemble des deux sites par rapport à $A$. gossypii. La phénologie des deux espèces au site de Kati reste cependant similaire, avec toutefois deux pics d'apparition pour l'espèce M. persicae (Figure 3a). On note vers la fin du cycle de la plante une diminution du taux d'infestation des pucerons. Par contre, à Sikasso, l'apparition de M. persicae et $A$. gossypii est faible au début, puis s'accroit considérablement en fin de végétation (Figure $3 \mathrm{~b}$ ).

\section{Détection des virus PVY et PLRV sur la pomme de terre}

Au total, 612 échantillons foliaires de pomme de terre ont été testés par ELISA. À Kati, $22,4 \%$ et $9,6 \%$ des échantillons se sont révélés positifs au PVY et au PLRV, respectivement, pendant la période considérée (Figure 4a). À Sikasso, des taux d'infection de $18,6 \%$ au PVY et de $8,1 \%$ au PLRV ont été déterminés (Figure $4 \mathrm{~b}$ ).

La présence des virus PVY et PLRV est détectée avec des taux importants sur l'ensemble des parcelles de pomme de terre dans les deux sites de production. L'incidence du PVY était relativement élevée de 2009 à 2011 aux deux sites, se situant entre 18,6 \% et $23,5 \%$, par rapport au PLRV pour laquelle elle varie entre $2,1 \%$ à $12,8 \%$ selon les années. L'analyse statistique conclut qu'il n'y a pas de différence significative quant aux taux moyens d'infection virale en fonction des années (Tableau 2) ni entre les sites (Tableau 3), qu'il s'agisse du PVY ou du PLRV.

Tableau 2. Taux moyen d'infection ( \pm écart-type) des échantillons de pomme de terre aux virus PVY et PLRV en fonction des années.

\begin{tabular}{lcc}
\hline Année & PVY & PLRV \\
\hline 2009 & $18,28 \pm 1,74 \mathrm{a}^{*}$ & $2,48 \pm 0,39 \mathrm{a}$ \\
2010 & $23,47 \pm 8,07 \mathrm{a}$ & $12,96 \pm 11,01 \mathrm{a}$ \\
2011 & $19,65 \pm 1,62 \mathrm{a}$ & $12,30 \pm 7,49 \mathrm{a}$ \\
& $\mathrm{F}=0,61$ & $\mathrm{~F}=1,16$ \\
& $\mathrm{P}=0,60$ & $\mathrm{P}=0,42$ \\
\hline
\end{tabular}

* Au sein d'une même colonne, les moyennes suivies d'une même lettre ne sont pas significativement différentes.

Tableau 3. Taux moyen d'infection ( \pm écart-type) des échantillons de pomme de terre aux virus PVY et PLRV en fonction des sites.

\begin{tabular}{lcc}
\hline Sites & PVY & PLRV \\
\hline Kati & $22,34 \pm 6,21 \mathrm{a}^{*}$ & $10,17 \pm 9,40 \mathrm{a}$ \\
Sikasso & $18,59 \pm 0,88 \mathrm{a}$ & $8,32 \pm 8,16 \mathrm{a}$ \\
& $\mathrm{F}=1,07$ & $\mathrm{~F}=0,07$ \\
& $\mathrm{P}=0,36$ & $\mathrm{P}=0,81$ \\
\hline
\end{tabular}

* Au sein d'une même colonne, les moyennes suivies d'une même lettre ne sont pas significativement différentes. 

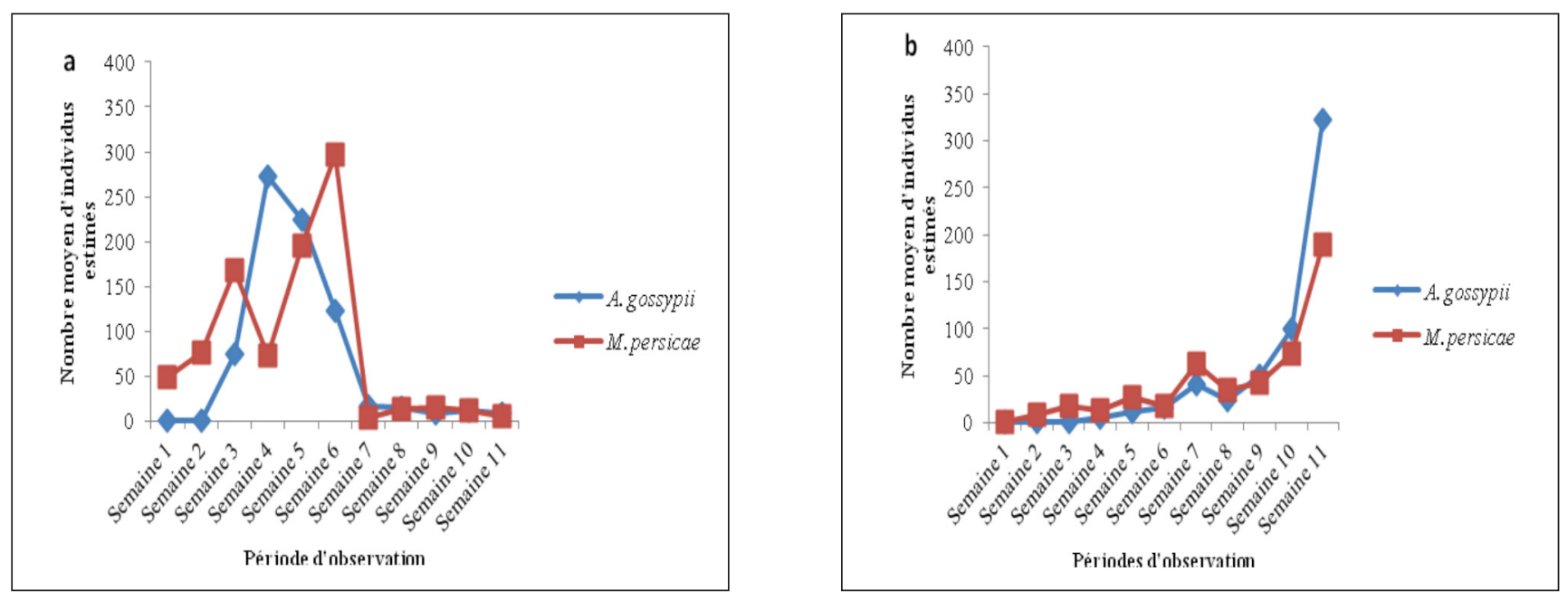

Figure 3. Phénologie de deux espèces de pucerons observées en cultures de pomme de terre de 2009 à 2011 à Kati (a) et Sikasso (b).
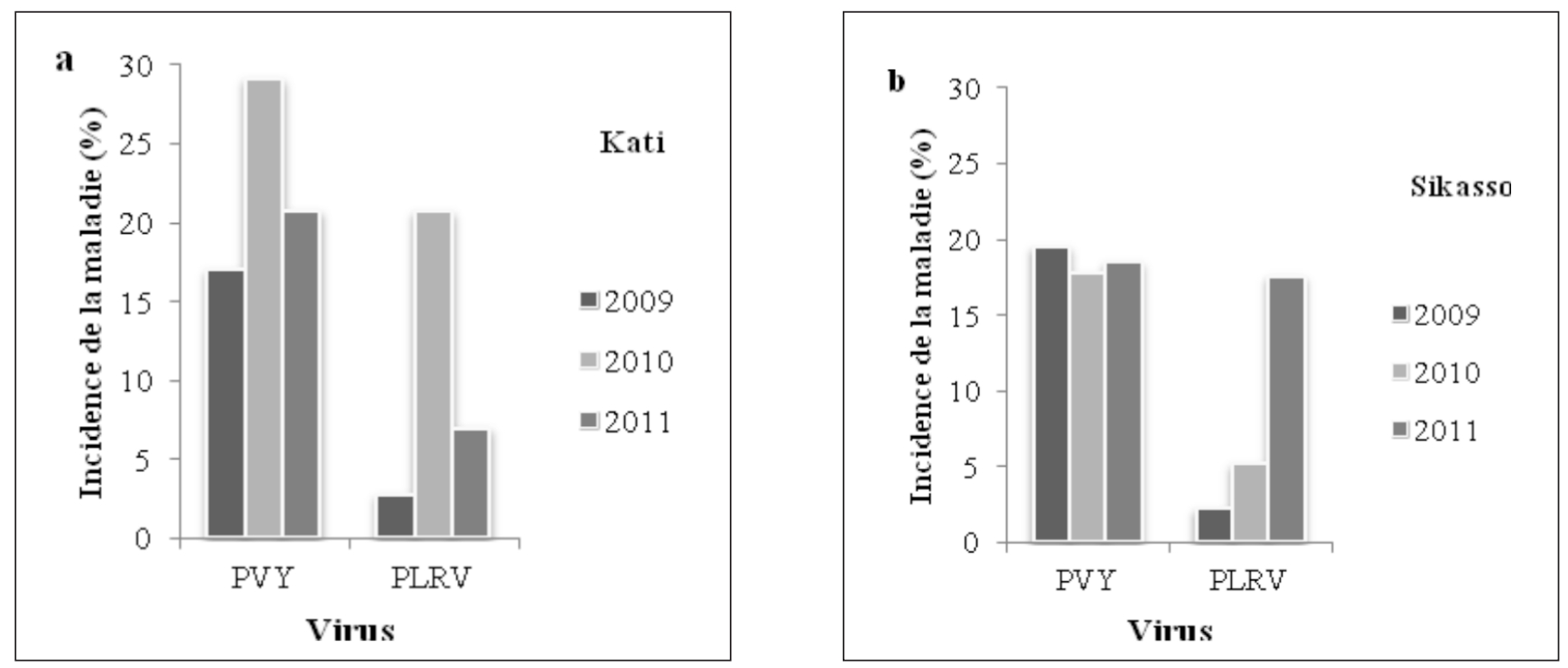

Figure 4. Incidence des virus PVY et PLRV sur la culture de pomme de terre de 2009 à 2011 à Kati (a) et Sikasso (b), au Mali.

\section{DISCUSSION}

Seulement six espèces de pucerons ont été recensées au Mali à ce jour (Remaudière et al. 1985). À partir des résultats de piégeage des pucerons ailés à l'aide de bacs jaunes installés dans des cultures de pomme de terre au Mali, 19 espèces de pucerons ont été recensées. Cette aphidifaune, caractérisée par un nombre important $(42,1 \%)$ d'espèces cosmopolites et par $10,5 \%$ d'espèces endémiques, a une forte affinité avec la faune subsaharienne. La plupart de ces espèces sont des ravageurs de cultures, dont l'espèce Myzus persicae, vecteur efficace notamment des viroses de la pomme de terre (Kennedy et al. 1962; Ragsdale et al. 2001; Robert et Bourdin 2001; Sigvald 1984) et d'une centaine de virus à de nombreuses autres cultures (Raman 1987).
Au sein des deux régions majeures de production de pomme de terre, l'espèce $L$. erysimi a été plus abondante à Kati $(69,7 \%)$ qu'à Sikasso $(5,5 \%)$. À l'inverse, $R$. rufiabdominalis domine dans les parcelles de Sikasso $(56,6 \%)$ comparativement à celles de Kati $(6,9 \%)$. Ensuite viennent les espèces $M$. persicae $(8,6 \%$ et $17,3 \%$ à Kati et Sikasso, respectivement) et $A$. gossypii $(7,7 \%$ et $10,4 \%$ à Kati et Sikasso, respectivement). Cette diversité d'espèces aphidiennes observée lors de nos investigations au Mali reste cependant faible si on se réfère à la diversité des pucerons à l'échelle mondiale. En effet, sur plus de 4700 espèces de pucerons répertoriées à travers le globe, 900 le sont en Europe (Hullé et al. 1999), dont environ 365 espèces de pucerons qui ont été identifiées en Belgique (Nieto-Nafria et al. 1999). En Afrique, 47 espèces aphidiennes ont été retrouvées 
sur 85 espèces végétales dans l'est algérien (Lamaari et al. 2010). Parmi les espèces de pucerons pour lesquels la pomme de terre fait partie du spectre d'hôtes, deux ont été observées au Mali, soit M. persicae et A. gossypii. Elles constituent des ravageurs importants de cette culture (Hullé et al. 1999). Ces espèces sont également des vecteurs importants de viroses chez des plantes hôtes comme le gombo (Abelmoschus spp.), le haricot (Phaseolus spp.), les cucurbitacées et les solanacées (Bordat et Arvanitakis 2004). La diversité des espèces de pucerons est un facteur important dans la dissémination des viroses que ces insectes sont susceptibles de transmettre, surtout pour les virus transmis de manière non persistante, comme c'est le cas pour le PVY chez la pomme de terre. Le nombre relativement élevé de pucerons capturés dans les pièges jaunes confirme que ces ravageurs sont problématiques dans les cultures de pommes de terre. Or, l'importance et la précocité de colonisation d'une culture par les ravageurs déterminent en grande partie l'ampleur des dégâts causés à la culture. Identifier les facteurs réduisant la fréquence et l'intensité des pullulations de pucerons en améliorant l'efficacité de leur contrôle naturel apparaît important pour mieux adapter les stratégies de protection des cultures. II faut cependant noter que des températures trop élevées peuvent conduire à une macération de certains pucerons en raison de la finesse de leur cuticule. Cependant, dans notre cas, les températures douces en cette période de l'année (novembre à mars) ont limité les pertes de matériel entomologique dans les pièges.

Le test ELISA a conduit à la détection de 49 réactions positives au PVY et 21 au PLRV, soit des taux d'infection aux deux virus de $22,4 \%$ et $9,6 \%$, respectivement, sur le total des échantillons testés pendant la période considérée. À Sikasso, le test ELISA a révélé $18,6 \%$ de taux d'infection au PVY et $8,1 \%$ pour le PLRV sur les 393 échantillons testés. Parmi les 612 échantillons testés à Kati, on note $28,4 \%$ de taux d'infection aux virus PVY et PLRV. Des taux plus élevés d'infection aux deux virus ont été enregistrés à travers le monde. Ainsi, jusqu'à $43,8 \%$ de la prévalence du PVY et du PLRV ont été constatés sur des échantillons foliaires et de tubercules de pomme de terre dans l'ouest du Cameroun (Njukeng et al. 2007). Une forte présence du PVY a été également rapportée chez la pomme de terre au Kenya (Ngugi 1983) et en Éthiopie (Yusuf 1988). En Belgique, le suivi de l'évolution des infections virales dans les parcelles de pomme de terre a permis de noter des fréquences élevées des virus PVY et PLRV, soit jusqu'à $79 \%$ et $34 \%$, respectivement (Rolot 2005).

À notre connaissance, il s'agit du premier travail réalisé sur la diversité et l'abondance des pucerons ainsi que sur la surveillance des virus associés en culture de pomme de terre dans les deux régions étudiées au Mali. Cependant, d'autres études sur l'incidence et la distribution des virus de la pomme de terre ont rapporté des infections de PVY (15,9\%) seul ou en infection mixte sur des Solanacées dans le nord du Bénin (Afouda et al. 2013) ou de PVY et PLRV dans le plateau de l'état du Nigeria avec plus de $30 \%$ de taux d'incidence de la maladie (Miha et al. 1993). Le puceron vert du pêcher ( $M$. persicae) demeure le vecteur le plus efficace de la majorité des virus transmis de façon non persistante, comme le PVY (Van Harten 1983; Verbeek 2010). Les processus de transmission persistante et non persistante sont à la base de la différence de spécificité de la relation virus-vecteur. En effet, durant la piqûre d'essai dans une plante infectée de virus, les virus non persistants viennent se fixer aux récepteurs spécifiques présents dans les pièces buccales du vecteur et seront relâchés par le biais des sécrétions salivaires lors d'un test ultérieur dans une autre plante que l'insecte désirera sonder (Gray 1996; Hogenhout et al. 2008). Les pucerons ailés en phase de dissémination ou de colonisation de nouvelles plantes sont particulièrement efficaces pour propager ce type de virus (Gray et Banerjee 1999). Contrairement au PVY, le PLRV est transmis selon le mode persistant et reste confiné dans les cellules du phloème. Une fois acquises par les pucerons, les particules virales passent par la lumière intestinale dans l'hémocèle et se diffusent dans I'hémolymphe ou elles peuvent rejoindre le canal salivaire avant d'être inoculées à une nouvelle plante (Marchoux et al. 2008). Cette transmission persistante se déroule quand le puceron se nourrit réellement sur la plante.

Bien que cette étude ait été menée pendant la saison sèche-fraîche, nous avons noté une relative abondance et une grande diversité des pucerons vecteurs. Les pratiques culturales, les changements climatiques et I'activité des pucerons vecteurs et de leurs plantes hôtes peuvent conduire à une large propagation des virus (Hanssen et al. 2010). La diversité des espèces de pucerons est donc un facteur important dans la dispersion de maladies virales que ces insectes sont capables de transmettre, en particulier pour les virus transmis de manière non persistante, comme le virus $Y$ de la pomme de terre. La détection de ces deux virus (PVY et PLRV) dans les champs de pomme de terre au Mali nécessite la prise de mesures de contrôle adéquates pour diminuer leur dispersion.

En conclusion, la mise en œuvre d'un plan de surveillance de l'aphidifaune dans les cultures comme celle de la pomme de terre est un élément essentiel afin d'identifier la diversité de vecteurs potentiels de viroses. En effet, à la suite de la collecte de ces données sur la diversité, l'abondance et la phénologie des espèces vectrices potentielles, des estimations des risques et des périodes de contrôle devraient permettre d'établir des modèles épidémiologiques et d'élaborer des stratégies de lutte comme des cultures intercalaires (intercropping) afin que les pucerons puissent décharger le PVY non persistant.

\section{RÉFÉRENCES}

Afouda, L.A.C., R. Kotchofa, R. Sare, V. Zinsou et S. Winter. 2013. Occurrence and distribution of viruses infecting tomato and pepper in Alibori in northern Benin. Phytoparasitica 41: 271-276.

Autrique, A. et L. Ntahimpera. 1994. Atlas des principales espèces de pucerons rencontrées en Afrique sub-saharienne. Administration Générale de la Coopération au Développement (AGCD), Publication agricole $n^{\circ} 33$.

Barbault, R. 1992. Écologie des peuplements: structure, dynamique et évolution. Éditions Masson, Paris, France.

Blackman, R.L. et V.F. Eastop. 2000. Aphids on the World's Crops: An Identification and Information Guide. 2nd ed. Wiley, Chichester, G.-B. 
Blackman, R.L. et V.F. Eastop. 2007. Taxonomic Issues. Pages 1-3 in H.F. van Emden et R. Harrington (éds.), Aphids as Crop Pests. CAB International, Cambridge, MA, É.-U.

Bordat, D. et L. Arvanitakis. 2004. Arthropodes des cultures légumières d'Afrique de l'Ouest, centrale, Mayotte et Réunion. CIRAD, Montpellier, France.

Clark, M.F. et A.N. Adams. 1977. Characteristics of the microplate method of enzyme-linked immunosorbent assay for the detection of plant viruses. J. Gen. Virol. 34: 475-483.

Coulibaly, M., D. Dembélé et B. Vanderhofstadt. 2002. Unité de production de plants de pomme de terre au Mali. Soc. Intl., Amatevi et IER.

EDES, 2012. Méthodes d'observation et d'échantillonnage au champ des populations de ravageurs. Surveillance et contrôle des bio-agresseurs dans les cultures. Cahier technique R.5: 29.

Fauquet, C. et J.C. Thouvenel. 1987. Plant viral diseases in the Ivory Coast. Documentation technique $n^{\circ} 46$. ORSTOM, Paris, France.

Gray, S.M. 1996. Plant virus proteins involved in natural vector transmission. Trends Microbiol. 4: 259-264.

Gray, S.M. et N. Banerjee. 1999. Mechanisms of arthropod transmission of plant and animal viruses. Microbiol. Mol. Biol. Rev. 63: 128-148.

Hogenhoot, S.H., E.D. Ammar, A.E. Whitfield et M.G. Redinbaugh. 2008. Insect vector interactions with persistently transmitted viruses. Annu. Rev. Phytopathol. 46: 327-359.

Hanssen, I.M., M. Lapidot et B.P.H.J. Thomma. 2010. Emerging viral diseases of tomato crops. Mol. Plant-Microbe Interact. 23: 539-548.

Harrington, R., S.J. Clark, S.J. Welham, P.J. Verrier C.H. Denholm, M. Hulle, D. Maurice, M.D. Rounsevell et N. Cocu. 2007. Environmental change and phenology of European aphids. Global Change Biol. 13: 1550-1564.

Hobbs H.A., D.M. Eastburn, C.J. D'Arcy, J.D. Kindhart, J.B. Masiunas, D.J. Voegtlin, R.S. Weinziert et N.K. McCoppin. 2000. Solanaceous weeds as possible sources of Cucumber mosaic virus in southern Illinois for aphid transmission to pepper. Plant Dis. 84: 1221-1224.

Hullé, M., E.T.A. Ighil, Y. Robert et Y. Monnet. 1999. Les Pucerons des plantes maraîchères. Cycles biologiques et activités de vol. INRA, Paris, France.

Jacky, F. et Y. Bouchery. 1982. Atlas des formes ailées des espèces courantes de pucerons. INRA, Colmar, France.

Kennedy, J.S., M.F. Day et V.F. Eastop. 1962. A Conspectus of Aphids as Vectors of Plant Viruses. Commonwealth Institute of Entomology, London, G.-B.

Laamari M., S. Tahar Chaouche, S. Benferhat, S.B. Abbès, H. Merouani, S. Ghodbane, N. Khenissa et P. Stary. 2010. Interactions tritrophiques: plante-puceron-hyménoptère parasitoïde observées en milieux naturels et cultivés de l'Est algérien. Entomol. faunistique 63: 115-120.

Leclant, F. 1999. Les Pucerons des plantes cultivées. Clefs d'identification. Tome II: Cultures maraîchères. Editions INRA, ACTA, Paris, France.

Marchoux, G., P. Gognalons et K.G. Sélassié. 2008. Virus des Solanacées. Du génome viral à la protection des cultures. Quae, Paris, France.

Mignon, J., P. Colignon, E. Haubruge et F. Francis. 2003. Effet des bordures de champs sur les populations de chrysopes [Neuroptera: Chrysopidae] en cultures maraîchères. Phytoprotection 84: 121-128.

Miha, A.M., H.W. Rossel et G.I. Atiri. 1993. Incidence and distribution of potato viruses in Plateau state, Nigeria. Afr. Crop Sci. J. 1: 131-138.

Ngugi, D.N. 1983. Potato production in Kenya: potentials and limitations [Solanum tuberosum]. Pages 140-142 in Research for Potato by the Year 2000. CIP, Lima, Peru.

Nieta-Nafria, J., G. Latteur, M.M. Durante, J. Tahon et N.P. Hidalgo. 1999. Les pucerons de Belgique (Hemiptera: aphididae). Parasitica $55:$ 5-38.
Njukeng, A.P., M.G. Chewachong, G. Chofong, P. Demo P. Sakwe et K.D. Njualem. 2007. Determination of virusfree potato planting materials by positive selection and screening of tubers from seed stores in the Western Highlands of Cameroon. Afr. Crop Sci. Conf. Proc. 8: 809-815.

Ragsdale, D.W., E.B. Radcliffe et C.D. Difonzo. 2001. Epidemiology and field control of PVY and PLRV. Pages 237-270 in G. Loebenstein, P.H. Berger, A.A. Brunt et R.H. Lawson (éds.), Virus and Virus-like Diseases of Potatoes and Production of Seed-potatoes. Kluwer Academic Publishers, Dordrecht, The Netherlands.

Raman, K.V. 1987. Transmission des virus de la pomme de terre par les insectes. Pages 11-16 in Centre international de la pomme de terre (éd.), La pomme de terre. Bulletins $\mathrm{d}^{\prime}$ information technique $\mathrm{n}^{\circ} 1$ à 19 . Centre international de la pomme de terre, Lima, Pérou.

Remaudière, G. et M. Remaudière. 1997. Catalogue des aphididae du monde. Éditions QUAE, Versailles, France.

Remaudière, G., V.F. Eastop et A. Autrique. 1985. Distribution des aphides de la région éthiopienne. Pages 77-93 in G. Remaudière et A. Autrique (éds.), Contribution à l'écologie des aphides africains. Organisation des Nations Unies pour l'Alimentation et l'Agriculture, Rome, Italie.

Robert, Y. et D. Bourdin. 2001. Aphid transmission of potato viruses. Pages 195-225 in G. Loebenstein, P.H. Berger, A.A. Brunt et R.H. Lawson (éds.), Virus and Virus-like Diseases of Potatoes and Production of Seedpotatoes. Kluwer Academic Publishers, Dordrecht, The Netherlands.

Rolot, J.-L. 2005. Analyse des facteurs régulant la dissémination du virus $Y$ de la pomme de terre (PVY) en vue de stratégies de lutte raisonnées. Thèse de doctorat, Faculté des Sciences Agronomiques de Grembloux, Gembloux, Belgique.

Sidibé, A., A. Berthé, B.M. Traoré, A.M. Dembélé et 0. Niangaly. 2008. Production de semences de pomme de terre dans la zone de I'Office du Niger au Mali. Atelier international APPRI 2008: Apprentissage, Production et Partage d'Innovations. Ouagadougou, Burkina Faso, 20-24 octobre 2008.

Sigvald, R. 1984. The relative efficiency of some aphid species as vectors of potato virus $\mathrm{Y}\left(\mathrm{PV} \mathrm{Y}^{\circ}\right)$. Potato Res. 27: $285-290$

Sikora, E.J., R.T. Gudauskas, J.F. Murphy, D.W. Porch, M. Andrianifahanana, G.W. Zehnder, E.M. Bauske, J.M. Kemble et D.F. Lester. 1998. A multivirus epidemic of tomatoes in Alabama. Plant Dis. 82: 117-120.

Taylor, L.R. 1981. Aphid Forecasting and Pathogens and a Handbook for Aphid Identification. Euroaphid, Rothamsted Experimental Station, Harpenden, G.-B.

Van Harten, A. 1983. The relation between aphid flights and spread of potato virus Y N (PVY N) in the Netherlands. Potato Res. 26: 1-15.

Verbeek, M., P.G.M. Piron, A.M. Dullemans, C. Cuperus et R.A.A. van der Vlugt. 2010. Determination of aphid transmission efficiencies for N, NTN and Wilga strains of potato virus Y. Ann. Appl. Biol. 156: 39-49.

Yusuf, A. 1988. Survey on potato and tomato viruses diseases in major growing areas. Pages 322-323 in S.P.L. Progress Report 1987/88. Ambo, Bako and Guder, Ethiopia. 